\title{
Beitrag zur Sectio caesarea auf Grund von 229 Fällen.
}

\author{
Von
}

\section{G. Leopold.}

Am 11. April 1905 war in der Klinik der 200. Kaiserschnitt ausgeführt wordẹn und damit ein neuer Anlass gegeben, auch das zweite Hundert zu durchmustern, wie es mit dem ersten Hundert im Jahre 1898 geschehen war $^{1}$ ). Andere, wichtigere Arbeiten traten aber hindernd in den Weg; die Erforschung des ferneren Geschicks der durch den Kajserschnitt gewonnenen Kinder brachte viele Verzögerungen mit sich; nicht zom Wenigsten lenkte aber die neuerstandene Hebotomie die Aufmerksamkeit mehr auf sich als auf den Kaiserschnitt, - jedenfalls zum grossen Vortheil der ganzen Sachlage.

Denn inzwischen wuchs die Zahl der Kaiserschnitte (bis Anfang März 1907) auf 229 heran; Hebotomien machten sich 48 (bis in die allerletzte Zeit 51) nothwendig. Gleichzeitig wurden aber alle anderen Operationen in der Therapie des engen Beckens sorgfältig weiter gepflegt, nämlich die künstliche Frühgeburt, die Wendung und Extraction und die Perforation, unter äusserster Einschränkung ihrer Anzeigestellung beim lebenden Kinde.

Hat doch der praktische Geburtshelfer für die Schwierigkeiten seines Berufes alles Anrecht darauf, an einem grossen, an engen Becken ausserordentlich reichen Material gezeigt zu bekommen, mit welchen Hülfsmitteln die Klinik das mütterliche und kindliche Leben retten kann, um sie unter Umständen selbst anzuwenden

1) Leopold und Haake. Dieses Archiv. Bd. 56. 
oder anwenden zu lassen. Er darf aber auch von der Klinik verlangen, dass sie ihm die Wege weist, wie er, auf sich allein angewiesen, in schwierigen Fällen am richtigsten und am humansten handelt, um so schonungsvoll wie möglich Mutter und Kind, aber namentlich die Erstere zu behandeln; um im kritischen Falle zum Wenigsten die Mutter, das Höchste, was der Mensch besitzt, zu retten.

Und wie in den vorausgegangenen Arbeiten ${ }^{1}$ ) offen dargelegt worden ist, welche Febler in der Anwendung aller der Operationen vorgekommen sind, die in der Therapie des engen Beckens das kindliche Leben retten sollen, so soll auch hier auseinandergesetzt werden:

1. ob und welche Fälle wir besser nicht dem Kaiserschnitt unterzogen hätten, und welche anderen Operationen heut zu Tage richtiger wären, mit anderen Worten, welche Todesfälle wir hätten vermeiden können, und

2. wie das weitere Geschick der Mütter und der Kinder war.

Zuvor aber möge sowohl über das erste, wie das zweite Hundert erst ein allgemeiner Ueberblick vorausgeschickt sein, welcher, namentlich für den Erfahrenen, eine Fülle des Interessanten in sich einschliesst.

In der Hauptsache (188 Mal) war die Enge des knöchernen Beckens die Veranlassung zu der grossen Operation. Die 12 anderen Fälle stehen aber mit der Verlegung des Beckens durch Geschwülste, Narbenverengung u. dgl. bezw. mit einer Geburtsunmöglichkeit in einem so innigen Zusammenhange, dass sie hier mit besprochen werden können.

Naturgemäss sind alle Fälle in die 2 Hauptgruppen: den erhaltenden und entfernenden Kaiserschnitt getrennt worden, da deren Anzeigestellung eine verschiedene ist ${ }^{2}$ ).

1) Siehe dieses Heft die Arbeiten der Herren Kannegiesser, Lichtenstein, Konrád, Meissner und Leisewitz.

2) Bei der Zusammenstellung des gesammten Materials und namentlich bei den Nachforschungen über das Schicksal von Mutter und Kind haben mich die früheren externen Hülfsärzte Herren DDr. Zinkeisen und Eisenberg, ferner einige meiner jetzigen Assistenten in dankenswerthester Weise unterstützt. 
704 Leopold, Beitrag zur Sectio caesarea auf Grund von 229 Fällen.

\section{Allgemeiner Ueberblick.}

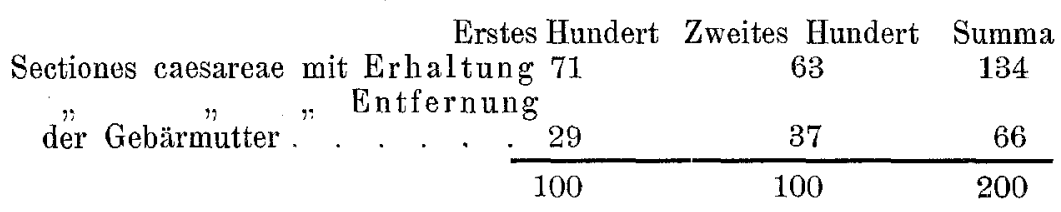

\section{Anzeige.}

1. Wegen Beckenenge.

Erstes Hundert Zweites Hundert
a) allgemein verengte Becken . . . . . . . 7
b) platt rhachitische Becken . . . . . . 11
c) allgemein verengt platt rhachitische Becken 65
d) rhachitisch schräg verengte Becken . . . 2
e) kyphotisch allgemein verengte Becken . . 4
f) aỉlgemein schräg verengte Becken . . . 2
g) osteomalacische Becken . . . . . . . 1
b) allgemein verengt pseudo-osteomalacische Becken
i) unregelmässig quer verengt platt rhachi- tische Becken . . . . . . . $\frac{\cdot-\frac{}{93}}{95}$

2. Aus andern Ursachen.

\section{Erstes Hundert.}

Geschwülste

Hernia uteri.

Ruptura uteri
$\mathrm{Z}_{\text {weites Hundert. }}$

Geschwülste . . . . . . . 2

Verjauchung. . . . . . . 1

Narbenstenose . . . . $\frac{2}{5}$

Grad der Beckenenge.

Erstes Hundert Zweites Hundert Summa (1 Becken nicht gemessen 1)

I. ${ }^{\circ}$ (Conj. vera $\left.9,5-8,5\right)$. . 0

II. $\left.{ }^{0}, ", 8,5-7,0\right) \cdot .24$

III. ${ }^{0} " \quad " 7,0-6,25$

37

2

III. " " bezw. 6,0

0) . 38

30

IV. ${ }^{\circ} \quad " \quad$ " $6,0-0$ )

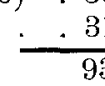

188 wegen Beckenenge.

Aus relativer Anzeige bei Beckenenge. 62 70

Aus absoluter Anzeige bei Beckenenge.

I. (Erhalt. S. c.) . . . . 20

II. (Entfern. S. c.) . . 
Anzeigen zur Sectio caesarea mit Entfernung des Uterus.

Verjauchung, Narbenstenose und

\section{Erstes Hundert Zweites Hundert Summa}

\section{Infection}

Enges Becken and Infection

Absolute Beckenenge . . . . 11

Enges Becken $u$. schwere Anämie 3

Enges Becken u.schwere Nephritis -

Enges Becken und schwerer Herz-

fehler . ...... . 2

Scoliot. bez. ankylot. Becken . 2

Atonie nach beabsichtigter erbaltender Sectio caesarea ${ }^{1}$ ) 3

Geschwülste $\left.{ }^{2}\right)$. . . . . . 4

Hernia uteri. . . . . . . 1

Ruptura uteri compl. bezw. incompl. . . . . . . . . 2

Enges Becken und schwere Blut. aus Riss im Lig. lat. . . .

Sterilisiruig auf Wunsch der

Frau und des Mannes bei hochgradiger Beckenenge und aus

verschieden. anderen Gründen -
$3 \quad 3$

45

$15 \quad 26$

$\overline{1}-3$

13

- 2

14

$-\quad 4$

13

11

Anzabl der Operationen in den einzelnen Jahren.

Erstes Hundert

Jahr

1882

1883

1884

1885

1886

1887

1888

1889

1890

1891

1892

1893

1894

1895

1896

1897

51

4.3

51

5

-

$-$
Erhalt. Entfer

Sa

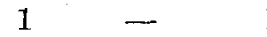

$1 \quad-\quad 1$

$3 \quad-\quad 3$

$\begin{array}{lll}1 & 2 & 3\end{array}$

$\begin{array}{lll}1 & 1 & 10\end{array}$

$5 \quad-\quad 5$

3

2

$\begin{array}{rrr}3 & 1 & 4 \\ 12 & 6 & 18\end{array}$

\begin{tabular}{rrr}
12 & 6 & 18 \\
8 & 9 & 17 \\
\hline 71 & 29 & 100
\end{tabular}

Zweites Hundert.

Erhalt. Entfern. Sa.

1898

1899

1900

1901

1902

1903

1904

1905

$\begin{array}{ll}10 & 10 \\ 37 & 66\end{array}$

1

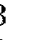

3

1

\begin{tabular}{rrr}
3 & 9 & $\mathbf{1 2}$ \\
6 & 2 & 8 \\
8 & 6 & $\mathbf{1 4}$ \\
6 & 6 & 12 \\
8 & 6 & $\mathbf{1 4}$ \\
14 & 4 & $\mathbf{1 8}$ \\
14 & 1 & $\mathbf{1 5}$ \\
4 & 3 & 7 \\
\hline 63 & 37 & $\mathbf{1 0 0}$
\end{tabular}

1) In je einem Falle lag noch Gonorrhoe bezw. Pseudoosteomalacie vor.

2) In Haake's Arbeit ist hier ein Versehen untergelaufen, dadurch, dass ein Fall zum Zwergwuchs gezählt worden. war. 
706 Leopold, Beitrag zur Sectio caesarea auf Grund von 229 Fällen.

$$
\text { Operateure. }
$$

I. Erhalt. S. c. Director: 51

9 Assistenten: 20

71

II. Entfern. S. c. Director: 27

2 Assistenten: 2

$\frac{29}{100}$
I. Erhalt. S. c. Director: 30

7 Assistenten: 33

63

4 Assistenten: 13

$\frac{37}{100}$

Daher: 132 Sect. caes. $=66$ pCt. (Director); 68 Sect. caes. $=34 \mathrm{pCt}$. kommen auf 17 Assistenten.

Verhältniss der Erst- zu den Mehrgebärenden.

Erstes Hundert.

I. Erhalt. S. caes. $27: 44=71$

II. Entfern. S. caes. $13: 16=\frac{29}{100}$

Insgesammt: $\overline{40: 60=100}$
Zweites Hundert.

I. $26: 37=63$

$53: 81=134$

II. $12: 25=\frac{37}{100}$

$=67 \mathrm{pCt}$.

$=33 \mathrm{pCt}$.

78 I parae: 122 Mehrgebärenden.

Wiederholter Kaiserschnitt.

Erstes Hundert.

Bei 13 Frauen.

Dreimal: bei 2 Frauen

(Nr. 9, 20, 37 u. 11, 35, 59)

Zweimal: bei 10 Frauen

(jedesmal in der Klinik)

bei 1 Frau, erst erhaltend, dann entfernend

Bei 13 Frauen. $\overline{38: 62=100}$

$$
\text { Von } 200 \text { bei } 31=15,5 \text { pCt. }
$$

Zweites Hundert

Bei 18 Frauen. Sa. bei 2 Frauen

4

bei 16 Frauen

26

(davon bei 8 die 2 S. caes.

entferuend)

Todesfälle.

Erstes Hundert.

Zweites Hundert.

I. Erhalt. S. caes. Von $71: 7=9,8$ pCt. I. Von $63: 4=6,35$ pCt.

II. Entfern. " " $\frac{29: 3=10,3}{100: 10=10}$ pCt. II. $\frac{37: 1=2,7}{100: 5=5 \text { pCt. }} "$

Summa: Von 200: $15=7,5$ pCt.

ad I, a) Nicht verschuldet:

Infection (No. 4)

Kyphoskoliose (15).

Gonorrhoe (69). 
Leopold, Beitrag zur Sectio caesarea auf Grund von 229 Fällen. 707

b) Auf Kosten der Klinik:

Nachblutung (27).

Mangelhafte Naht und Handcompression (34).

Besser hochgradige Atonie (45). war $\{$ Gonorrhoe (67).

Porro:

ad II. a) Nicht verschuldet:

Medullarsarkom (20).

Zwergin, schwerer Herzfehler (29).

b) Auf Kosten der Klinik:

Nachblutung (18). b) Auf Kosten der Klinik:

Peritonitis (83).

Sepsis (?). Herzfehler (85).

Riss in den Darm (121).

Incarceration des versenkten retrofl. Uterus, Peritonitis (129).

ad II. a) Nicht verschuldet:

Septisch übernommen (56).

b) -

Erstes Hundert.

Zweites Hundert.

Summa.

Auf Kosten der Klinik:

$\begin{array}{ll}\text { I. } 4: 68=5,8 \text { pCt. } & \text { I. } 4: 63=6,35 \text { pCt. } \quad 8: 131=6,1 \text { pCt. }\end{array}$

$\begin{array}{lll}\text { II. } 1: 27=3,7, \quad \text { II. } 0: 36=0 \quad n & 1: 63=1,5 \%\end{array}$

$5: 95=5,2 \mathrm{pCt} \quad 4: 99=4 \quad \mathrm{pCt}$.

9 Todesfälle auf 194 Kaiserschnitte $=4,6$ pCt., die der Klinik zur Last fallen.

Todesfälle nach Jahren geordnet.

Die ersten 12 Jahre: 1882-1893: 54 mit 3 Todesfällen $=5,5 \mathrm{pCt}$. zweiten $12 "$ 1894-1905: $140 \quad$ "6 " $=4,2 "$

Todesfälle nach Sectio eaesarea nur aus relativer Anzeige bei Beckenenge; der Klinik zur Last fallend:

$62: 3$ $70: 2$

$132: \overline{5}$ Todesfällen $=3,7 \mathrm{pC}$.

Verlauf des Wochenbettes (mit Weglassung der Todesfälle).

Erstes Hundert.

1. Fieberfrei .....6 60

2. Leichte Steigerung . . 19

3. Septische Erkrankung 9

Eklampsie und Arthr. gonorrh. ...... 1

Psychose . . . . 1

(十10 Todesf.)
$Z_{\text {weites }}$ Hundert.

Summa

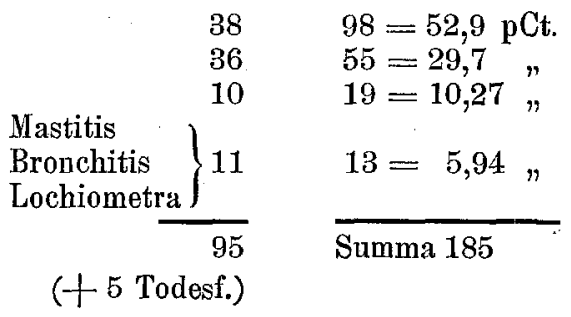


708 Leopold, Beitrag zur Sectio caesarea auf Grund von 229 Fällen.

$$
\text { Ergebnisse für die Kinder. }
$$

Erstes Hundert.

Zweites Hundert.

Summa

I. Erhalt. S. caes. Alle Von 63: 62 lebend geb.

71 lebend geb.

II. Entfern. S. caes. Von Von 37: 30 Jebend geb.

29 Kind. 23 leb. geb.

$$
94 \text { leb. geb. }
$$

ad I. $\left\{\begin{array}{c}\text { In den } \\ \text { nä̈chsten } \\ 3 \text { Woch. } \dagger\end{array}\right.$

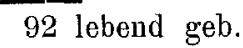

$134: 183$

7

Demnach: todtgeboren 6

später gest. 7

lebend entl. 87

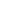

ad I. 1 unt.d.Geb. 3 später $\uparrow$ ad II. 6 vor der Geb. abgestorben 1 bald darnach $66: 53$

100<smiles>C=C</smiles>

Nachforschung über das spätere Schicksal der Kinder. Beim 2. Hundert.

I. Erhalt. S. caesarea. (No. 72-134) 63 Kinder.

Ohne Nachricht . $\overline{16}$

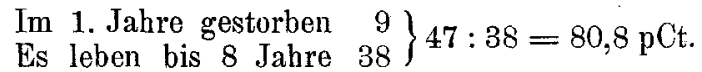

63

II. Entfern. S. caesarea. (No. 30-66) 37 Kinder.

Todt geboren. . 6

Bald gestorben . . . 2

Ohne Nachricht . . 6

Im 1. Jahre gestorben 6$\} 23: 17=73,9 \mathrm{pCt}$. 37

Nehmen wir endlich vom 3. Hundert die bisher erfolgten 29 Kaiserschnitte hinzu, so gruppiren sie sich folgendermaassen: 
Leopold, Beitrag zur Sectio caesarea auf Grund von 229 Fällen. 709

I. Erbalt. S. caesarea.

Anzeige.

Absolute

Beckenenge.

9
Relative

Beckenenge.

12

II. Entfern. S. caesarea.

\begin{tabular}{|c|c|c|c|c|}
\hline & 4 & 4 & 8 lebend & $\begin{array}{l}7 \text { lebend. } \\
1 \text { vorh. } \dagger .\end{array}$ \\
\hline Sa. & 13 & 16 & 27 genesen & 28 Iebend. \\
\hline
\end{tabular}

Mütier.

19 lebend

$2+$

Alle 21 lebend.

Alle 229 Fälle zusammengenommen, mit Berechnung der Todesfälle, welche der Klinik zur Last fallen:

9 Todesfälle auf 194 Kaiserschnitte (s. oben)

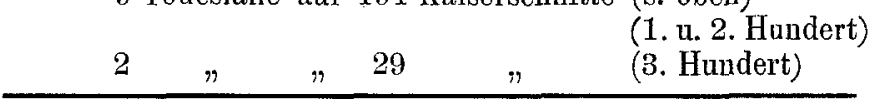

11 Todesfälle auf 223 Kaiserschnitte

$=4,9$ pCt. mütterliche Mortalität.

Das vorliegende Material enthält eine derartige Fülle von Beobachtungen und Erfahrungen, dass man ein Buch schreiben müsste, wenn man sie alle erschöpfen wollte. Dazu findet sich vielleicht später die geeignete Veranlassung.

Jetzt gilt es, die operative Therapie beim engen Becken zur Rettung des kindlichen Lebens möglichst vollständig zu besprechen, weshalb sich an die in diesem Heft befindlichen Arbeiten meiner Assistenten über die Hebotomie, die künstliche Frühgeburt, Wendung and Extraction, Perforation und Zange noch der Kaiserschnitt zu schliessen hat.

Selbstredend müssen uns hierbei die Todesfälle der Mütter in erster Linie interessiren und es gilt die Frage zu beantworten:

Erstens: $O b$ und welche Fälle besser durch ein anderes Verfahren erledigt worden wären, mit anderen Worten: Welche Todesfälle zu vermeiden waren?

Beschäftigen wir uns hier mit dem 2. Hundert 1 ) und den letzten 29 Operationen des 3. Hunderts, so kommen 6 Todesfälle in Betracht, welche auf jeden Fall der Klinik zur Last fallen. Bd. 56 .

1) Betreffs des ersten Hundert s. Leopold und Haake. Dieses Archiv. 
710 Leopold, Beitrag zur Sectio caesarea auf Grund von 229 Fällen.

1. Frau L. 14. 3. 1900 . J.-No. 383.

$29 \mathrm{j}$. I para hatte ein hochgradig allgemein verengt platt rhachit. Becken mit Zeichen schwerer Rhachitis am ganzen Körper. Sp. 23, 23,5, 32,5, 16.5, Conj. diag. 9, Conj. vera $7 \mathrm{~cm}$.

Wehen seit vier Stunden kräftig. Kreuzbein im unteren Theile scharf abgeknickt. Doppelter Vorberg. Linea ganz abtastbar. Muttermund für 2 Finger durchgängig. Blase gesprungen. Placenta hinten.

Der sehr harte Kopf steht pendelnd über dem Beckeneingang. Missverbältniss $z$ wischen beiden auffallend. Eintreten oder Configuration des Kopfes erscheint ganz ausgeschlossen. Die Eheleute sprechen den Wunsch nach einem lebenden Kinde aus.

Daher Sectio caes. aus relativer Anzeige im Einverständniss mit dem Ehepaar und nach genauer Darlegung des Sachverhalts: Spontane Geburt liess sich bestimmt ausschliessen. Perforation des lebenden Kindes wäre unbegründet gewesen. Die Hebotomie wurde damals noch nicht in der Klinik ausgeführt.

Operation am 14. 3. 1900, 5 Uhr Nachm. Oper.: Verfasser.

Beim Vorwälzen des Uterus aus der Bauchhöhle und beim Senkrechtstellen desselben vor dem Uterusschnitt, was selbstverständlich, wie immer, sehr rorsichtig erfolgte, entstand rechts hinten und unten in der Gegend des inneren Muttermundes, jedenfalls durch Abreissen einer Bauebfellverlöthung mit der Nachbarschaft ein Peritonealriss, welcher durch 7 feine Seidennähte geseblossen wurde. Darmach typische Operation mit 10 tiefen und 16 oberflächlichen Seidenkropfnähten.

Mädchen, leicht asphyktisch, nach 5 Minuten wieder belebt, $49 \mathrm{~cm}$ lang, $2800 \mathrm{~g}$ schwer. Kopfdurchmesser: gerader 11, senkrechter 9, grosser querer $8,5 \mathrm{~cm}$. Grösster Umfang $35,5 \mathrm{~cm}$.

Es bedarf wohl keiner Darlegung, dass, wie immer, alle Vorsichtsmaassregeln der Asepsis zur Anwendung gekommen waren.

Nur zweierlei muss hervorgehoben werden: Ca. $31 / 2$ Stunden vor der Operation hatte der Assistent, welcher dann bei der Operation half, die einzige innere Untersuchung, die doch nothwendig war, vorgenommen. Gummihandschuhe brauchten wir damals noch nicht. Und 2. Die Secrete der Scheide der Frau waren, wie es seit Jahren geschieht, vorher untersucht und ohne Gonokokken gefunden worden. Aber bei der Kürze der Zeit hatte eine erschöpfende Secretuntersuchung an 10 bis 20 Präparaten Ieider nicht erfolgen können.

Schon am 3. Tage p. op. setzte hohes Fieber ein, 39,6 Temp. Im Collumsecret wurden Streptokokken nachgewiesen. Vom 12. Tage an rechtsseitige Pleuropneumonie, welche am 30 . Tage zum Tode fübrte.

Section: Croupöse Pneumonie der rechten Lunge, rechtsseitige, fibrinöse, eitrige Pleuritis, sept. Milatumor, Verlöthung des Uterus vorn. Vereiterung der Stichcanäle; sept. Endometritis; Metro-Thrombophlebitis, Thrombose der 1. Uterina und Iliaca.

Es kann keinem Zweifel unterliegen, dass die Klinik diesen Todesfall verschuldet. Aber alle Erörterungen und Untersuchungen haben, wie es so häufig geschieht und wie man ja auch in den Berichten aus anderen Kliniken Aehnliches lesen kann, die Ursache der Infection nicht mit Sicherheit anfgedeckt.

Der Fall lehrt wiederum, dass die Sectio caesarea, weil Lapa- 
rotomie, in ihrem Ausgange, trotz aller Maassnahmen der Antisepsis und Asepsis, unberechenbar bleibt und die Prognose ausnahmslos vorsichtig zu stellen ist.

Das neugeborene Mädchen war bei der letzten Nachforschung am 1. 3. 04 vier Jahre alt, gesund und frisch, aber: es hatte keine Mutter!

Jetzt würde man in solchem Falle, bei Aussichtslosigkeit des Abwartens, in der Klinik die Hebotomie machen und grössere Aussicht haben, Mutter und Kind durchzubringen.

In der Privatpraxis wäre ebenfalls, bei Erfüllung aller Vorbedingungen, die Hebotomie das Richtigste. Sind diese aber nicht erfüllt, würde dann der praktische Geburtshelfer einen Fehler begehen, wenn er schliesslich zur Perforation des vielleicht noch lebenden Kindes greift und würde Jemand mit Recht einen Stein auf ihn werfen? Ich glaube es nicht.

Ganz ähnlich liegen die Verhältnisse im zweiten Falle:

2. Frau W., 12. 6. 00. J.-No. 931. 31 jähr. I para. Allgemein verengt platt-rhachitisches Becken. 26,$5 ; 24 ; 29 ; 16,5 ; 8,5$; Vera 6,5 . Mit 12 Jahren Typhus. Im 25. Jahre Gelenkrheumatismus. Jetzt Mitralinsufficienz.

Geburtsdatier bis zur Operation 28 Stunden. Kolpeuryse zur Schonung der Fruchtblase.

Innere Untersuchung eine halbe Stunde vor der Operation: Muttermund 3 markstückgross. Blase vor der Operation gesprengt. Nichteintreten des sehr grossen Kopfes.

Operation (Verf.) 4 Uhr Nachm.: 9 tiefe und 11 oberflächliche Seidenknopfnähte. Verlauf normal.

Knabe asphyktisch, nach 20 Min. wiederbelebt, später noch längere Zeit stark cyanotisch. $51,5 \mathrm{~cm}$ lang, $3570 \mathrm{~g}$ schwer. Grösster querer Kopfdurchmesser $10,25 \mathrm{~cm}$, kleiner querer $9 \mathrm{~cm}$; horizontaler Kopfumfang $34 \mathrm{~cm}$, grösster Umfang $39 \mathrm{~cm}$.

Bei der Mutter trat am 4. Tage zuerst starke Athemnoth, dann Trachealrasseln und starker Husten bei Dämpfang auf der rechten Brusthälfte auf. Zeichen von Peritonitis fehlten. Unter Zunahme dieser Erscheinungen trat bei höcbster Temp. von 39,6 am 7. Tage der Tod ein. Sektion verweigert.

Pneumonie? Sepsis?

Die Klinik nimmt auch diesen Fall auf sich. Erschwerend war hier in hohem Grade die Mitralinsufficienz. Entbunden werden musste die Fran. Weiteres Abwarten auf Spontangeburt wäre bei Conj. vera von $6,5 \mathrm{~cm}$ ein Unrecht gewesen.

So käme nur in Frage, ob zur damaligen Zeit die Sectio caesarea zur Rettung des Kindes, aber mit Entfernung des Uterus, nicht die schnellste und noch sicherere Operation gewesen wäre. Und doch konnte durch die auch hiermit verbundene Narkose 
712 Leopold, Beitrag zur Sectio caesarea auf Grund von 229 Fällen.

die Mitralinsufficienz so ungünstig beeinflusst werden, dass sie ebenfalls zum Tode geführt hätte.

Nach alledem bleibt dieser Todesfall, als durch die Operation bedingt, zu Recht bestehen.

Heutzutage käme die Pubotomie in Frage. Nur haben viele weitere Erfahrungen erst noch klar zu legen, ob bei einer Vera von $6,5 \mathrm{nach}$ der Pubotomie das Becken so gespreizt werden darf, dass ein Kopf mit 10,25 cm Querdurchmesser und horizont. Kopfumfang von $34 \mathrm{~cm}$ schadlos für Mutter und Kind (sei es spontan, sei es operativ) das Becken passiren kann.

Das Neugeborene wurde vom Vater abgeholt und in Pflege gegeben, woselbst es bald gestorben ist.

Der dritte Todesfall beruht auf einer bei der Operation nicht bemerkten Verletzung des Colon transversum, in Folge von schweren Verlöthungen nach früherer Sectio caesarea:

3. Frau C. geb. Eb., 9. 2. 04.29 jähr. IV para. Platt-rhachit. Becken: 25, 26, 31, 17, 81/2,61/2 Vera. Seit 12 Stunden in der Geburt.

1. Geburt 27. 3. 97. Sectio caesarea. Am 20. Tage mit Kind entlassen. Kind nach $11 / 2$ Jabren gestorben.

2. Geburt 27. 7. 02 . Vorfall der Nabelschnur. Absterbendes Kind. Perforation und Kranioklasie.

Im Wochenbett am 3. Tage Temp. 38,8, Puls 76. Gonokokken nachgewiesen.

Pat. kommt am Ende der 3. Schwangerschaft zur Klinik. Wehen seit 12 Stunden.

Innere Untersuchung 5 Stunden vor der Operation: Muttermund 3 markstückgross. Blase steht. Wird kurz vor der Operation gesprengt.

Operation (Oberarzt Dr. Kannegiesser): Die Bauchschnittnarbe ist in ihrem unteren Theile am Uterus adhärent; oben etwa in Wallnussgrösse herniös vorgebuchtet. Bauchschnitt rechts von der Narbe. Eröffnung der Bauchhöhle sehr erschwert durcb viele Adhäsionen zwischen Netz, Uterus und Bauchwand. Doppelte Unterbindung. Umschneidung der Hautadhäsionsstelle und Excision der Bauchhernie. Uterus mit 7 tiefen und 14 oberfächlichen Seidenknopfnähten wieder geschlossen.

Knabe $48 \mathrm{~cm}$ lang, $2600 \mathrm{~g}$ schwer. Grosser querer Kopfdurchmesser $83 / 4 \mathrm{~cm}$, horizontaler Umfang $34 \mathrm{~cm}$.

Die ersten beiden Tage relatives Wohlbefinden der Operirten.

Am 3. Tage Meteorismus. Temp. 38,1.

Am 4. Tage grosse Unruhe, hoher Meteorismus. Temp. 39,1, Puls 150. Plötzlicher Verfall. Tod.

Section: Peritonitis mit Austritt von Gas und Koth in die freie Bauchböhle aus einem groschengrossen, mit platten Rändern versehenen Loch in der Wand des Quercolons. 
Dieser Unglïcksfall belastet die Klinik. Beim Lösen der Adhäsionen ist trotz aller Vorsicht, jedenfalls unbemerkt, die Verletzung erfolgt; vielleicht auch beim Herauswälzen des mit der Umgebung stark verlötheten Uterus, ähnlich wie im vorhergehenden Falle beim Heranswälzen ein Peritonealriss passirte.

Das Kind gedieh an Ammenbrust sehr gut; starb aber im 3. Jahre an Brechdurehfall.

Die schweren Adhäsionen, welche bei der wiederholten Sectio caesarea oft viel Zeit zur Beseitigung in Anspruch nehmen und schon mit der Verlängerung des ganzen Eingriffs Mutter und Kind sehr schädigen können, legen die Frage nahe, ob es hier nieht richtiger gewesen wäre, das relativ kleine Kind $(48 \mathrm{~cm}$ lang, $2600 \mathrm{~g}$ schwer) lieber durch Porro zu entwickeln, um dann den Uterus zu entfernen. Maassgebend hätte hier der Umstand mit sein können, dass die Frau schon genug durchgemacht, ausserdem im 2. Wochenbette sich gonorrhoisch inficirt erwiesen hatte. Letztere Erkrankung hätte auch, nach unseren sehr traurigen Erlebnissen ${ }^{1}$, die Vornahme der Hebotomie, als sehr unvortheilhaft für die Wunde, ausgeschlossen.

Nach alledem kann in solchem Falle, beim Zusammentreffen mehrerer ungünstiger Verhältnisse, selbst die Klinik berechtigt sein, die Perforation des lebenden Kindes in Betracht zu ziehen und abzuwägen gegen einen Porro. Um wieviel mehr der praktische Geburtshelfer, falls er in solehem Falle ganz auf sich allein angewiesen sein sollte?

Bei dem vierten Todesfall (No. 129) handelte es sich um einen ganz eigenthümlichen Befund: Incarceration des versenkten retroflectirten Uterus und Ileus.

M. H., 32 jähr. II para, war am 25. November 1895 nach Vorfall der Nabelschnur durch Perforation des todten Kindes entbunden worden. Allgemein verengt platt rhachitisches Beeken: 22, 22, 30, 161/2; $8 \frac{1}{2}$; Vera $6^{3} / 4-7 \mathrm{~cm}$. Relative Anzeige. Geburtsdauer bisher 15 Stunden. Innere Untersuchung in der Klinik 5 Stunden vor der Operation. Portio verstrichen. Muttermund dreimarkstück gross. Kopf tritt nicht ein. Künstlicher Blasensprung.

Operation 7 Uhr $28 \mathrm{Min}$. Vorm. (Verf.) 7. 11. 04. Uterus genäbt mit 9 tiefen und 14 oberfächlichen Seidenknopfnähten.

$\mathrm{Kn} .50 \mathrm{~cm}$ lang, $3550 \mathrm{~g}$ schwer. Grosser querer Kopfdurchmesser $91 / 2 \mathrm{~cm}$, horizontaler Umfang $37 \mathrm{~cm}$.

1) s. Kannegiesser, Hebotomie No. 5 bei bestehender Gonorrhoe mit nachfolgender sehr schwerer Erkrankung im Wochenbett. 
714 Leopold, Beitrag zur Sectio caesarea auf Grund von 229 Fällen.

Am 3. Tage des Wochenbettes Meteorismus, Erbrechen. Temp. 38,9. Puls 96. Keine Flatus.

Am 5. Tage nach Einfübrung eines Darmrohres Abgang wässrigen Stuhles und von Luft. Temp. 38,5. Puls 130.

$\mathrm{Am}$ 6. Tage hochgradiger Meteorismus. Da als Ursache des $\mathrm{Zu}$ standes nach dem ganzen bisherigen Verlaufe Darmabknickung anzunehmen ist, wird der Leib wieder aufgemacht.

Darnach zeigt sich, dass die Darmschlingen enorm gebläht sind, das Colon aber contrahirt ist. Peritoneum stark injicirt und mit feinen weisslichen Anflagerungen versehen. Der Uterus ist retroflectirt und im kleinen Becken incarcerirt. Seine Naht wird erst sichtbar, nachdem die Gebärmutter aufgerichtet worden ist. Zwei mittlere Seidenuähte sind aufgegangen; die Wunde ist zum Theil mit gelblich weissen Auflagerungen bedeckt. Im kleinen Becken blutig schmierige Massen. Nach Entfernung derselben wird der Uterus schnell abgetragen und sein Stumpf im unteren Wundwinkel fixirt. Es tritt aber bald darnach der Tod ein.

Section: Acute diffuse Peritonitis. Das Kind erbielt in der Klinik eine Amme und wurde am 12. Tage mit einer Gewichtszunahme von $40 \mathrm{~g}$ gegenüber dem Anfangsgewicht entlassen. Nach 2 Monaten ist es in auswärtiger Pflege gestorben.

Die Beurtheilung dieses Falles lehrt:

1. dass das Abwarten einer spontanen Geburt falsch gewesen wäre; und

2. dass zur Rettung des kindlichen Lebens schon damals (7. 11. 04) die Hebotomie am Platze, jedenfalls richtiger gewesen wäre, als der Kaiserschnitt.

Was im vorliegenden Falle zur Entzündung der Naht, dann zur Incarceration und schliesslich zur Peritonitis führte, war mit grösster Wahrscheinlichkeit folgendes:

Wir hatten schon bei manchen Kaiserschnitten beobachtet, dass es, wie bei jeder anderen Wöchnerin, auch einmal zu einer Lochiometra mit mässigem Fieberanstieg kommen kann. Dann half am schnellsten, unter denkbar grösster Vorsicht, eine Einstellnng der Portio, vorsichtiges Anziehen der vorderen Collumwand, unter Spülıng mit warmer, physiologischer, steriler Kochsalzlösung und Ablassen des verhaltenen Wochenflusses.

Leider geschah dies hier nicht; welche Gründe dagegen sprachen, lässt sich nachträglich nicht mehr beurtheilen. Man darf aber annehmen, dass die Stauung des Wochenflusses zur Dehnung der Naht und schliesslich zur Entzündung tührte, die dann bald die Verlöthung des retroflectirten Uterus, seine Incarceration und die Bauchfellentzündung verursachte.

Wir kommen schliesslich zu den beiden Todesfällen unter den ersten 29 Kaiserschnitten des dritten Hundert. 
Die 19 jähr. H. W., kam am 6. 10. 1905 zur Geburt. Allgemein verengt platt rhachit. Becken; 25, 26, 33, 17;8, 5; Conj. vera 6, $5 \mathrm{~cm}$. Geburtsdauer 10 Stunden, heftige Wehen. Eintreten des Kopfes ganz ausgeschlossen.

Innere Untersuchung 3 Stunden ante op. Muttermund für 2 Finger durchgängig. Blase steht. Linea ganz abtastbar. Vorberg scharf und spitz hereinspringend. Secrete in Bezug auf specifische Keime unsicher.

Operation (Verf.) am 6. 10. 05, Abends 9 Uhr 30 Min. 8 tiefe und 21 oberflächliche Seidenknopfnähte.

Knabe, $53 \mathrm{~cm}$ lang, $3350 \mathrm{~g}$ schwer. Grosser, querer Kopfdurchmesser $9 \mathrm{~cm}$. Horizontaler Umfang $35 \mathrm{~cm}$.

Schon am 1. Tage p. op. setzt hohes Fieber ein, welches unter den Zeichen der Peritonitis am 5. Tage zum Tode führte.

Von der Uterusnaht wurden nach erfolgter Section. verschiedene Stücke herausgeschnitten und nach sorgfältiger Härtung mikroskopisch untersucht. Alle Schnitte liessen nur die eine Annahme zu, dass die Todesursache eine gonorrhoische Endometritis gewesen war.

Nachträglich ist der Fall wohl folgendermaassen zu beurtheilen:

Bestehende Gonorrhoe bleibt unter allen Umständen ungünstig, ja verhängnissvoll für eine Hebotomie und eine Sectio caesarea. Dies haben uns nun schon so viele Fälle gelehrt, dass an der Wahrheit dieses Satzes nicht mehr gezweifelt werden kann.

Da hier Spontangeburt ausgeschlossen und das Kind lebensfrisch war, so konnte die Fragestellung nur so sein: Kann man das Kind noch retten, ohne die Mutter wesentlich zu schädigen? Die Antwort lautet: In der Klinik wäre das Beste der Porro gewẻsẹn. In der Privatpraxis, falls der Arzt ganz auf sich allein gewesen wäre, das' Beste die Perforation des lebenden Kindes.

In dem sechsten (letzten) Todesfall konnte mit Sicherheit nachgewiesen werden, dass im Drange ausserordentlich vieler sich häufender und sehr schwerer Fälle in der Klinik ein Fehler in der Asepsis untergelaufen war.

Frau E. B., geb. B., war am 10. 7. 06, früh, zur Klinik gebracht worden. 28j. Ipara. Kindliches Becken, 21, 23, 29, 16,5, 8. Vera 6. Absolute Anzeige. Ende der Schwangerschaft. Starke Wehen.

Zwei Stunden vor der Operation innerlich untersucht (Handschuhe). Portio verstrichen. Muttermund 2 Markstückgross. Blase steht. Linea überall abtastbar.

Operation (Verf.) 10. 7. 06, Vorm. 10 Uhr. Fruchtwasser meconiumhaltig. Uterus genäht mit 8 tiefen und 12 oberflächlichen Seidenknopfnäbten.

Mädchen, leicht asphyktisch, $49 \mathrm{~cm}$ lang, $3300 \mathrm{~g}$ schwer. Grosser querer Durchmesser des Kopfes $9^{1 / 2} \mathrm{~cm}$, horizontaler Umfang $36 \mathrm{~cm}$. 
716 Leopold, Beitrag zur Sectio caesarea auf Grund von 229 Fällen.

Vom 3. Tage an unter Singultus hohes Fieber. Am 5. Tage 39,9, Puls 128. Linke Uteruswand sehr schmerzhaft. Leib nicht aufgetrieben. Bauchwunde reactionslos. Am 6. Tag 40,5, Puls 146. Links zunehmende Leibschmerzen. Meteorismus. Tod.

Section: Eitrige Peritonitis. Infection der Uteruswunde. Beim Auftrennen der Nabt findet sich zwischen mittlerem und unterem Drittel der. Operationswunde eine ziemlich starke Vene, von einem eitrig schmelzenden Thrombus erfüllt. Auch in der Umgebung thrombosirte, mit Eiter gefüllte Thromben. Uteruswand daselbst sulzig durchtränkt. Die inficirte Partie liegt im Wesentlichen links von der Wunde und reicht bis zur linken Uternskante. Tuben frei von Eiter. Parametrien stark ödematös.

Die Nachforschungen über das Zustandekommen dieser bei der Operation erfolgten Infection ergaben mit voller Sicherheit, dass sie nur auf diejenige Person zurückgeführt werden konnte, welche als die einzige, bei der Operation direct betheiligte, unterlassen hatte, Gummihandschuhe anzuziehen. Jedenfalls konnten nach Allem, was vorausgegangen war, ihre zwar sorgfältig gewaschenen und desinficirten, aber nicht mit Handschuhen versehenen Hände als absolut sicher nicht angesehen werden.

Somit ist auch jede weitere Betrachtung über die Art der Entbindung überflüssig. Die Sectio caesarea war indicirt, die erhaltende Methode die gegebene. Traurig genug, dass die Mutter das Leben des Kindes, welches zur Zeit über ein halbes Jahr alt und ganz gesund ist, mit dem Tode büsste.

Ueberblicken wir noch einmal diese 6 Todesfälle, um die Nutzanwendung daraus zu ziehen, so fallen sie alle auf Rechnung der Klinik.

Jetzt würde aber im ersten Falle der Kaiserschnitt durch die Hebotomie zu ersetzen und dadurch mit grosser Wahrscheinlichkeit die Mutter am Leben zu erhalten sein.

Im $z$ weiten Falle wäre es besser gewesen, den Porro anzuwenden.

Der dritte Fall mahnt zur allergrössten Vorsicht bei Lösung der Adbäsionen nach einem früheren Kaiserschnitt. Da in diesem Falle die Gebärende noch dazu gonorrhoisch war, so wäre bei Abwägung aller Verhältnisse der Porro die beste Methode gewesen, das Kind zu retten und die Mutter gesunden zu lassen.

Im vierten Falle wäre jetzt die Hebotomie die gegebene Operation.

Im fünften Falle, der durch Gonorrhoe complicirt war, wäre 
bei fast absoluter Beckenenge die Porro'sche Operation noch der vorsichtigste Eingriff gewesen.

Der sechste Fall mahnt auf das Eindringlichste, vor jedem Kaiserschnitt, da or eine Laparotomie ist, noch einmal alles Material und alle hülfereichenden Personen auf das Gewissen zu prüfen, ob sie thatsächlich den Anforderungen der Asepsis genügen.

Hier wird aber wohl für alle Zeiten der fromme Wunsch hinter dem Erreichbaren etwas zurückbleiben.

Zum Schluss möge mit wenigen Worten des späteren Schicksals der Kinder gedacht sein, welche durch den Kaiserschnitt zur Welt kamen.

Die Nachforschungen über das zweite Hundert ergaben, dass nach dem erbaltenden Kaiserschnitt nur über 47 von 63 Kindern etwas zu erfahren war. Von diesen 47 Kindern lebten $38=80,8$ pCt. bis za 8 Jahren, also 9 gingen im 1. Jahre zu Grunde; eine Procentziffer, welche den allgemeinen Erfahrungen über die Sterblichkeit im ersten Lebensjahre entspricht.

Weniger günstig lauten die Erfahrungen nach dem Porro'schen Kaiserschnitt; doch kann hier recht wohl die Kleinheit der Zahlen mit daran Schuld tragen.

Hier war von 37 Kindern nur über 23 eine Nachricht zu gewinnen und zwar waren von diesen 23 nur 17 bis zu 8 Jahren $=73,9$ pCt. am Leben geblieben.

Also insgesammt von 70 Kindern des zweiten Hundert lebten bis zu 8 Jahren noch $55=78,5$ pCt. 